\section{Cureus}

Received 06/09/2014

Review began 06/10/2014

Review ended 07/22/2014

Published 07/22/2014

\section{(๑) Copyright 2014}

Cvek et al. This is an open access article distributed under the terms of the Creative Commons Attribution License CC-BY 3.0., which permits unrestricted use, distribution, and reproduction in any medium, provided the original author and source are credited.

\title{
Cardliac Radiosurgery for Malignant Ventricular Tachycardia
}

\author{
Jakub Cvek ${ }^{1}$, Radek Neuwirth ${ }^{2}$, Lukas Knybel ${ }^{3}$, Lukas Molenda ${ }^{3}$, Bretislav Otahal ${ }^{3}$, Jakub \\ Pindor $^{2}$, Mária Murárová ${ }^{2}$, Michal Kodaj ${ }^{2}$, Martin Fiala ${ }^{2}$, Marian Branny ${ }^{2}$, David Feltl ${ }^{1}$ \\ 1. Department of Oncology, University Hospital Ostrava 2. Department of Cardiology, Podlesí Hospital \\ Třinec, CZECH REPUBLIC 3. Department of Oncology, University Hospital Ostrava, CZECH REPUBLIC
}

$\square$ Corresponding author: Jakub Cvek, jakub.cvek@fno.cz

Disclosures can be found in Additional Information at the end of the article

\section{Abstract}

Ventricular tachycardia is a frequent cause of mortality after myocardial infarction. Current treatment includes the implantation of cardioverter defibrillators and adjunctive therapies, such as catheter ablation or cardiac surgery. In patients where standard treatment fails, preclinical data showed that radiosurgery ablation of the ectopic substrate might be a viable option. Authors present a case report of cardiac radiosurgery in a patient with malignant ventricular tachycardia. The stereotactic radiosurgery system, CyberKnife, was used; the applied dose was $25 \mathrm{~Gy}$ in one fraction. Within the follow-up period of 120 days, no signs of toxicity were noted and no episode of malignant arrhythmia has been detected. This case report demonstrates that stereotactic radiosurgery of recurrent ventricular tachycardia after inefficient catheter ablation might be a viable option for patients unsuitable for cardiosurgical intervention. Further research on this topic is highly warranted.

Categories: Cardiology, Radiation Oncology

Keywords: cyberknife, ventricular tachycardia, cardiac radiosurgery

\section{Introduction}

Ventricular tachycardia (VT) is a re-entrant rhythm that causes significant morbidity and mortality after myocardial infarction or other cardiac disease [1]. Implantable cardioverter defibrillators (ICDs) offer excellent protection from sudden death; however, adjunctive therapies are often necessary to reduce shocks in ICD recipients [2]. Catheter ablation is often used as primary therapy with success rate in the range of $53-67 \%$ [3]. There are a big variety of reentrant circuits, involving subepicardial, subendocardial, and intramural reentry pathways [4], so radiofrequency (RF) ablation is not always successful and new approaches are highly warranted. One of the possible solutions is the use of high-energy photons [5].

Extracranial stereotactic ablative radiotherapy with the robotic linear accelerator CyberKnife has demonstrated high precision in the dose delivery with low toxicity profile [6]. Moreover, the so-called "functional radiosurgery" (radiosurgery ablation of specific functional areas) is widely used in the brain and its high efficacy has been proven [7]. These data make a good theoretical basis for the expansion of functional radiosurgery beyond traditional indications, e.g. trigeminal neuralgia. Previous pre-clinical studies have documented the feasibility of this approach [5].

In our case report study, we describe methodology, toxicity, and results of radiosurgery ablation for patient with malignant arrhythmia. 


\section{Cureus}

\section{Case Presentation}

\section{Patient history}

A 72-year-old female with recurrent ventricular tachycardia and recurrent arrhythmic storm had been treated for more than 10 years for dilated cardiomyopathy with ejection fraction of the left ventricle of $25 \%$ and mitral regurgitation Grade III/IV. After the episode of syncope, the ICD-Cardiac Resynchronization Therapy (ICD-CRT) was implanted in January 2013. She was treated with beta-blockers in maximal, well-tolerated dose (amiodarone was contraindicated due to long QT-interval). Her coronary arteries showed only mild coronary stenosis. ECG monitoring reported very frequent ventricular extrasystoles. During repeated electrophysiology studies, VT of the same morphology was induced in the form of spontaneous premature ventricular complexes (PVCs). Catheter RF ablations (both endocardial and epicardial) were ineffective due to excessive thickness of the myocardium. Because of repetitive arrhythmic storms, other treatment options were evaluated. She was not a candidate for cardiac surgery due to severe comorbidity, such as systemic hypertension, paroxysmal atrial fibrillation, diabetes mellitus, chronic pulmonary obstruction disease, and chronic renal dysfunction. As all standard treatment options were exhausted, she became a candidate for stereotactic radiosurgery.

The treatment was approved by local IRB and Ethics Commission. The patient signed the informed consent.

\section{Stereotactic radiosurgery ablation}

Arrhythmogenic ectopic substrate localization was based on two electrophysiology (EP) studies using an electroanatomic-mapping system (CARTO, Biosense-Webster, Israel). The first EP study was endocardial in the left ventricle (transseptal access); the second EP study was combined endo- and epicardial mapping (epicardial mapping through dry pericardiocenthesis with added subxiphoidal access). The source of arrhythmia was localized at the base of lateral wall of left ventricle. Electroanatomic maps of CARTO were registered with diagnostic CT scans (Figure 1).

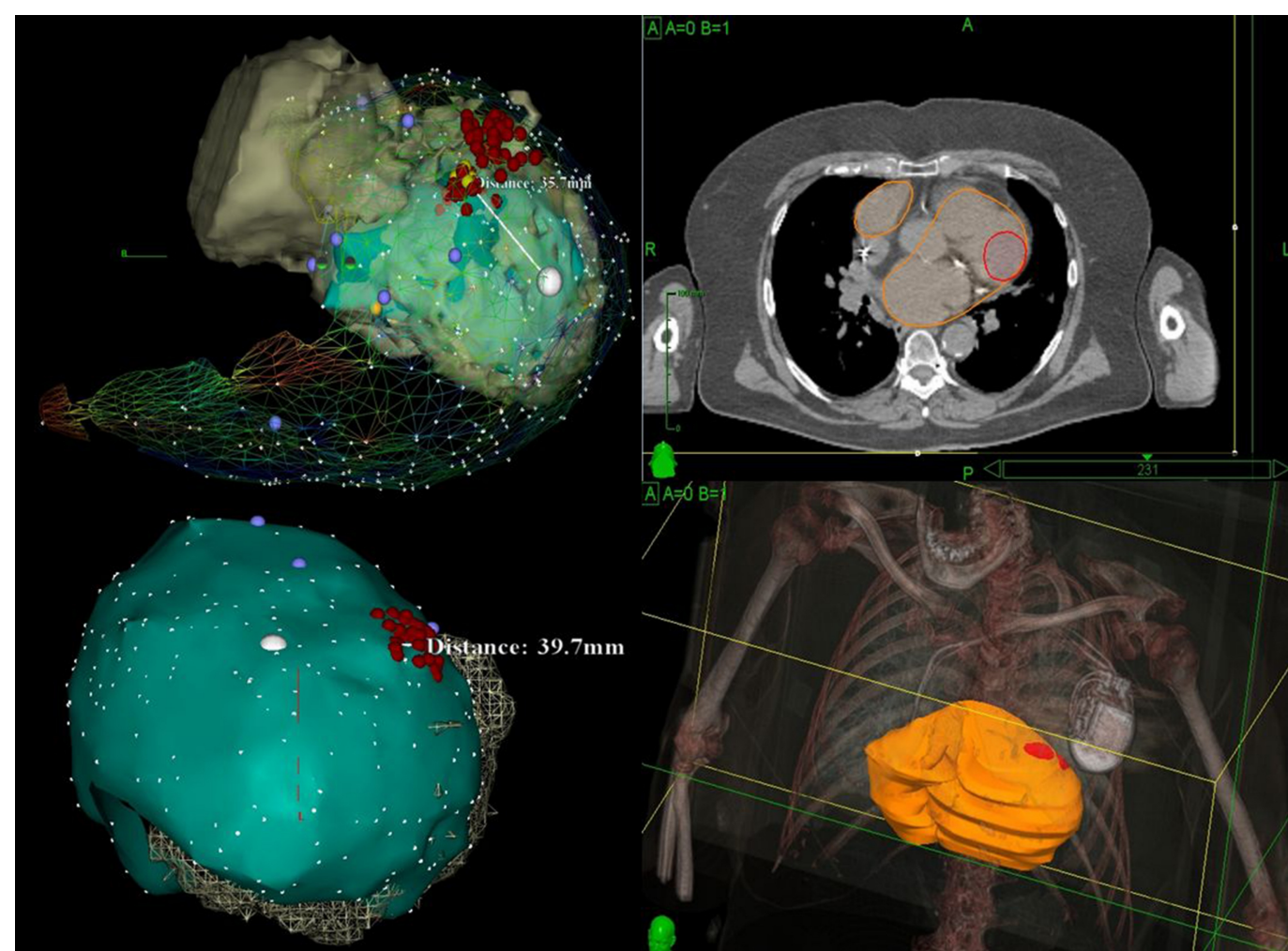




\section{Cureus}

\section{FIGURE 1: Image registration of CARTO and CT with the}

localization of the ectopic substrate

Clinical target volume (CTV) included ectopic lesion (the source of arrhythmia) during systole and diastole (internal target volume, ITV); no additional margin for planning target volume (PTV) was added because of high conformity index $(1,27)$ and low average correlation error (Figure 2).
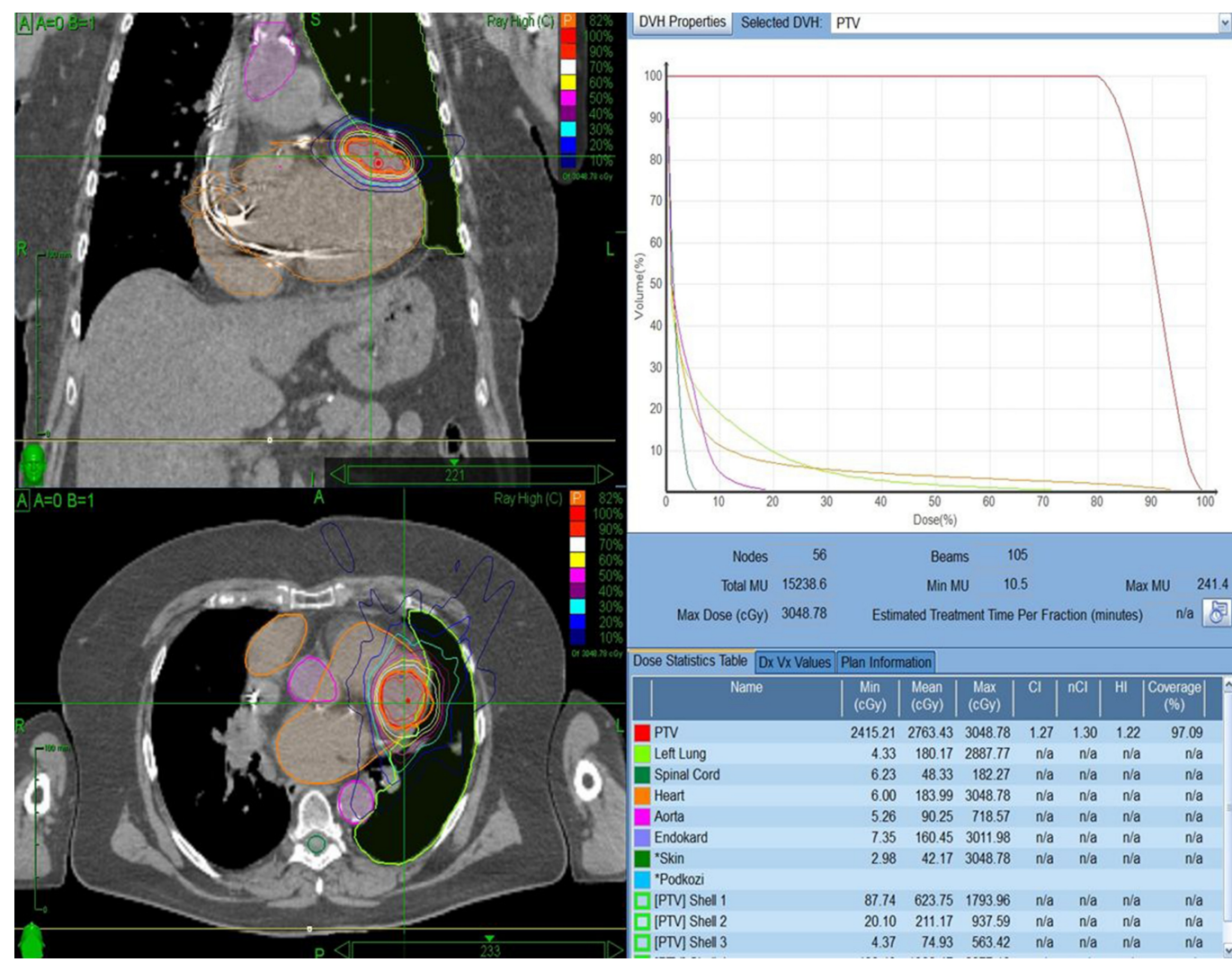

\section{FIGURE 2: Isodose plan and dose-volume histogram}

LV electrode of the stimulation system in the lateral branch of coronary sinus was used as fiducial marker for respiratory tracking method (Synchrony) (Figure 3). 


\section{Cureus}

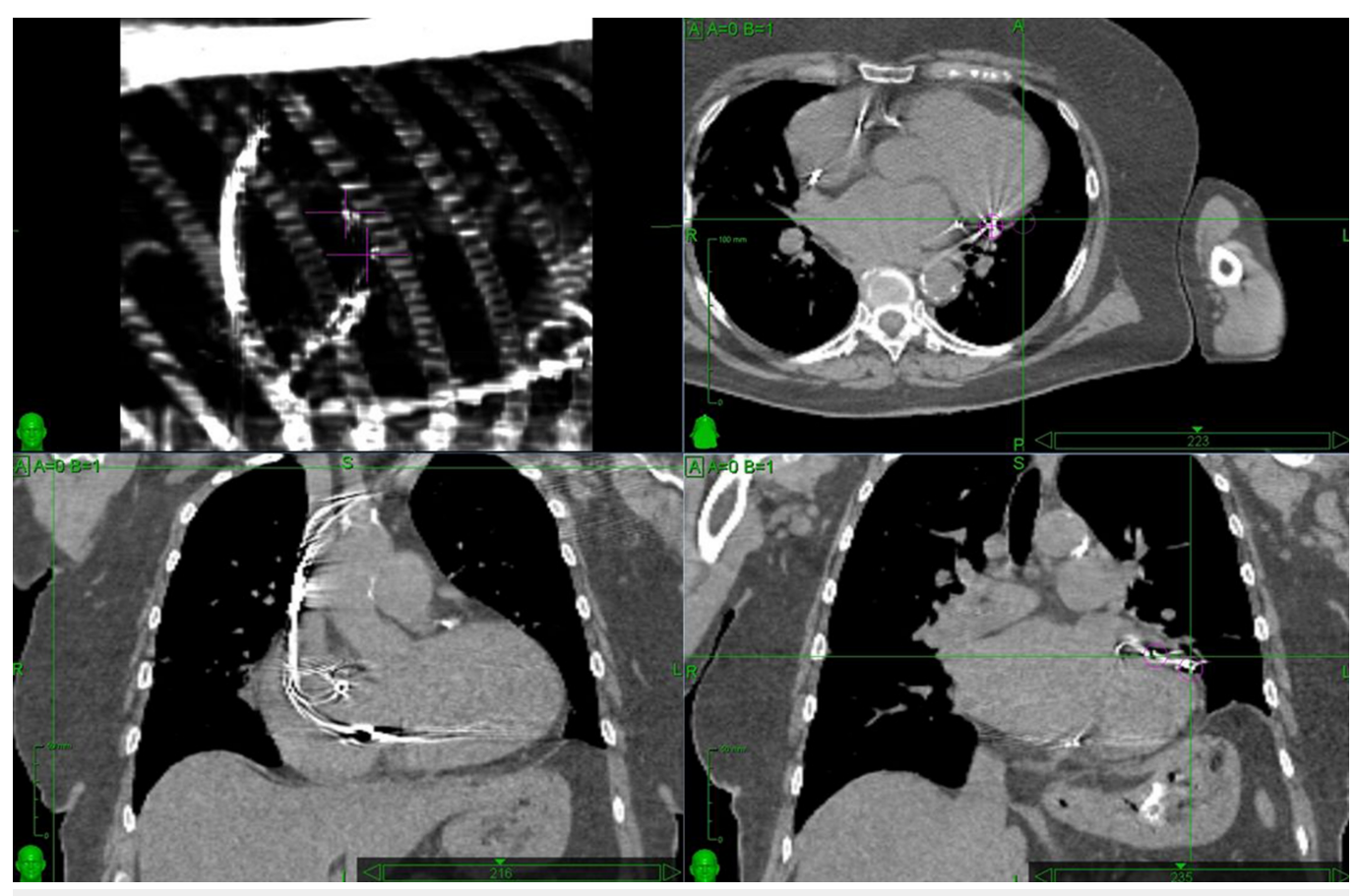

FIGURE 3: LV electrode of the stimulation system used as fiducial marker for real-time tracking

The motion of the target was controlled with the Synchrony system that allows real-time tracking. Position of implanted fiducial was extracted from the treatment X-ray images and correlated with the breathing light signal from external markers on the patient's chest. The correlation model was made before the start of the treatment, updated after each $\mathrm{x}$-ray acquisition, and adapted continuously during the radiosurgery treatment.

Multiplan treatment planning system, version 3.5 with sequential optimization, and CyberKnife radiosurgery system, version 8.5, were used (both Accuray, Inc., Sunnyvale, CA). The prescribed dose was $25 \mathrm{~Gy}$ in one fraction. Metal deletion technique (MDT) was used for the evaluation how artifacts from leads affect the dose distribution; the difference was lower than $1 \%$. Monte Carlo dose calculation was used for evaluation close proximity to lung tissues effected dose the distribution and difference was lower than $3 \%$.

Continual monitoring was performed under the auspices of cardiologists.

\section{Results}

Precision Reached

One hundred and five beams and 56 nodes were used. Dosimetric parameters were as follows: conformity index 1.27, new conformity index 1.3, coverage $97 \%$, and homogeneity index 1.22 . Median dose was 27.6 Gy (range 24.2-30.5 Gy); prescribed isodose line was 82\%. Treatment time was 114 minutes. Nine correlation models had to be created, median one per 12 minutes (range 2-15 min), to reach precision better than $3 \mathrm{~mm}$ (average correlation error 1,1 $\mathrm{mm}$ ). 
After the end of session, the ECG analysis according to ICD memory was performed and no ventricular tachycardia was found. Ten days after the radiosurgery session, the number of PVCs decreased from $9-10 \%$ to $1-3 \%$, and non-sustained ventricle tachycardias (nsVT) diminished as documented in ECG monitoring during hospitalization and during repetitive ambulatory ECG Holter monitoring (Figure 4).

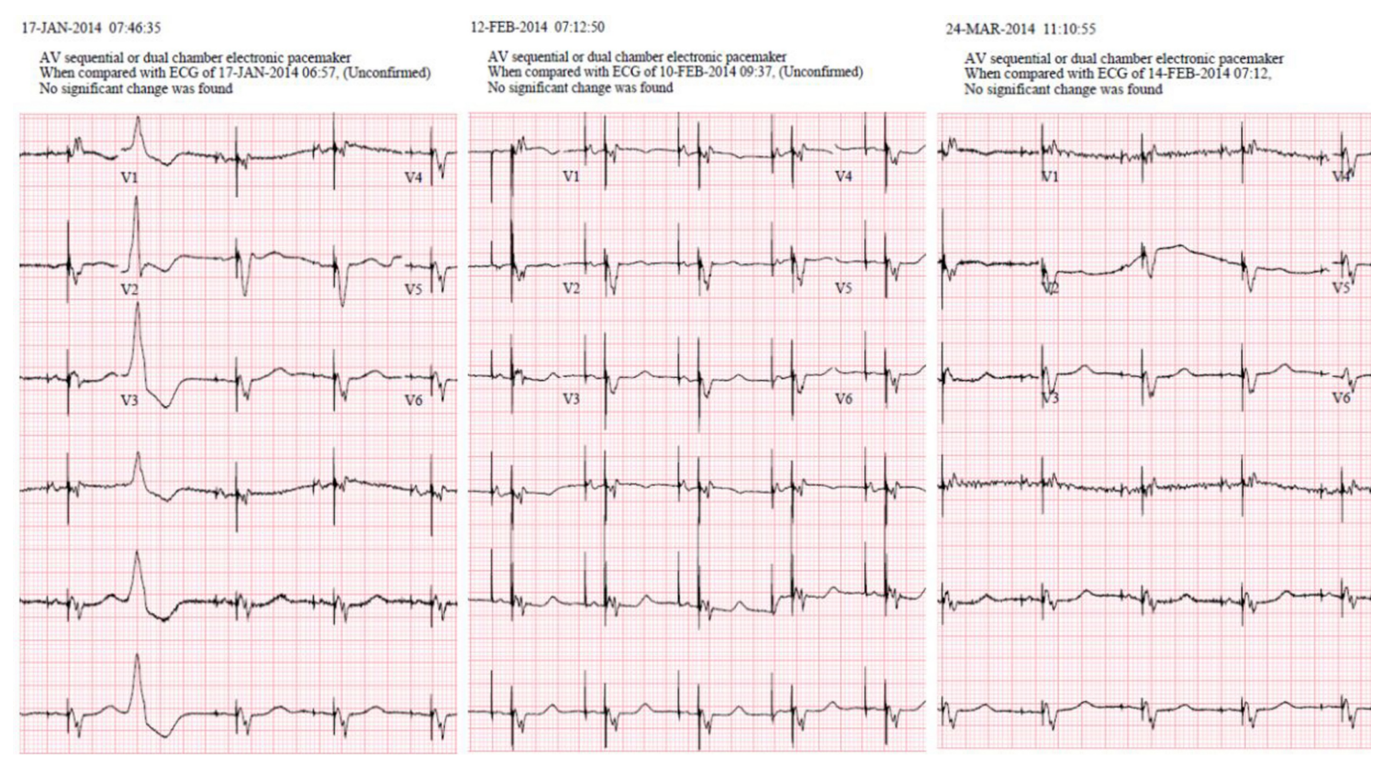

\section{FIGURE 4: ECG before the treatment (left), two weeks (middle), and two months (right) post-treatment}

In the subsequent follow-up of 120 days, no episode of malignant arrhythmia has been detected by ICD.

\section{Toxicity}

Ten days post-treatment, only minimal elevation of troponin $\mathrm{T}$ serum level was detected (0.024-0.033). After six weeks, no complications or side effects were found. There were no signs of radiation pneumonitis nor pericardial effusion.

\section{Discussion}

Malignant ventricular arrhythmia is a life-threatening condition [1], and the implantation of ICD is definitely a life-saving procedure. However, quality of life is not always ideal in these patients [2] and other therapeutic procedures are necessary, such as pharmacotherapy and catheter ablation. Catheter ablation based on electrophysiology monitoring is successful in approximately $50 \%$ of patients; improvement at the level of $25 \%$ might be reached with the addition of epicardial catheter [9]. Failure of this approach makes an indication for cardiac surgery [10], but not all patients can be operated upon due to comorbidities. For this subgroup of patients, no further treatment option has been available. Functional radiosurgery with highenergy photons was tested in an experimental study with encouraging results [5]. Since the heart is moving continuously and thoracic anatomy is very complex, the treatment system must be very precise and able to track moving targets. The CyberKnife radiosurgery system, with its capability for motion tracking, seems ideal for this purpose [11].

The most important step in the treatment planning is the identification of the ectopic substrate. 
Unfortunately, our system does not allow direct registration of pictures from electroanatomical mapping (CARTO) and computed tomography. So the target was localized with indirect comparison of relative distances with electrode of the stimulation system as the orientation point. This workflow is very time-consuming and prone to errors so direct registration of the above-mentioned imaging methods with the ability to contour precisely the ablation volumes would be extremely helpful.

The patient was treated with the respiratory tracking system, Synchrony, for maximum organ sparing. We used a stimulating electrode as a fiducial marker, which proved suitable, but cannot serve as universal solution. A more sophisticated alternative is the implantation of classical fiducial markers (gold seeds) to the myocardium. This procedure requires training and some experience, since in heavily pre-treated patients, every additional invasive procedure might be risky. To assure precision better than $3 \mathrm{~mm}$, nine correlation models had to be created, which is quite a high number. This was due to a very irregular breathing pattern in a seriously ill patient. For maximum conformity, sequential optimization planning was used and the difference between CTV and ITV made only 14\%. Better conformity could be reached using ECG-gating; this might be necessary in patients that have normal heart movements so the target moves much more than in our patient who suffered from cardiomyopathy.

Dose prescription is an important aspect of radiosurgery. The dose of 25 Grays was adopted from the first human case report by Lo, et al. [8]; this dose was considered safe, and safety was our highest priority. Dose escalation above $25 \mathrm{~Gy}$ (30 Gy or more) leads to scar formation so the treatment might be more effective, as reported by Blanck, et al. [12]. All in all, the dose issue is one of the most interesting points in cardiac radiosurgery; we believe that our paper speeds up the discussion and moves this extremely promising area of functional radiosurgery a step forward.

The goal of the treatment is full elimination of malignant arrhythmia. In our case, 10 days after the radiosurgery session, the number of PVCs decreased from $9-10 \%$ to $1-3 \%$ and non-sustained ventricular tachycardias (nsVT) diminished as documented by ECG monitoring during a hospital stay and then with subsequent repetitive ambulatory ECG Holter monitoring. Moreover, no episode of malignant arrhythmia has been detected by ICD during the four-month post-treatment monitoring. In general, our results are in accordance with Lo, et al., who reported significant reduction in non-supraventricular tachycardia (NSVT) episodes after radiosurgical ablation, possible reduction in total treated VT/VF episodes, no shocks delivered to treat VT/VF episodes, and improvement of patient's quality of life [8].

Ten days after the therapy, only a minimal rise of troponin $\mathrm{T}$ serum level was detected (0.0240.033). No other toxicity was found. After six weeks, systolic function of LV has improved. This confirms the low toxicity profile that was published previously [8]; neither cardiac nor pulmonary complications were reported. Obviously, these data only create hypotheses and must be confirmed in a properly designed Phase I clinical trial. The safety goal of stereotactic radiosurgery might be to have a lower complication rate than the one of epicardial ablation. In a study reported by Sacher, et al., epicardial ablation was necessary in 121 of 913 ablative procedures for VT (13\%); acute and delayed major complications were reported in 5\% and 2\% of patients, respectively [9].

\section{Conclusions}

Stereotactic radiosurgery of recurrent ventricular tachycardia after inefficient catheter ablation seems a viable option for patients unsuitable for cardiosurgical intervention.

\section{Additional Information}




\section{Disclosures}

Human subjects: Consent was obtained by all participants in this study. Local IRB and Ethics Commission issued approval. Conflicts of interest: In compliance with the ICMJE uniform disclosure form, all authors declare the following: Payment/services info: All authors have declared that no financial support was received from any organization for the submitted work. Financial relationships: All authors have declared that they have no financial relationships at present or within the previous three years with any organizations that might have an interest in the submitted work. Other relationships: All authors have declared that there are no other relationships or activities that could appear to have influenced the submitted work.

\section{Acknowledgements}

This work was supported by Institutional support - RVO-FNOs/2014

\section{References}

1. Moss AJ, Greenberg H, Case RB, Zareba W, Hall WJ, Brown MW, Daubert JP, McNitt S, Andrews ML, Elkin AD; Multicenter Automatic Defibrillator Implantation Trial-II (MADIT-II) Research Group: Long-term clinical course of patients after termination of ventricular tachyarrhythmia by an implanted defibrillator. Circulation. 2004, 110:3760-5.

2. Nazarian S: CMR for mapping the missing dimension in ventricular tachycardia ablation . JACC Cardiovasc Imaging. 2010, 3:286-8. 10.1016/j.jcmg.2009.11.007

3. Sacher F, Tedrow UB, Field ME, Raymond JM, Koplan BA, Epstein LM, Stevenson WG: Ventricular tachycardia ablation: Evolution of patients and procedures over 8 years . Circ Arrhythm Electrophysiol. 2008, 1:153-61. 10.1161/CIRCEP.108.769471

4. Stevenson WG, Khan H, Sager P, Saxon LA, Middlekauff HR, Natterson PD, Wiener I: Identification of reentry circuit sites during catheter mapping and radiofrequency ablation of ventricular tachycardia late after myocardial infarction. Circulation. Oct;(4 Pt 1):. 1993, 88:1647-70.

5. Sharma A, Wong D, Weidlich G, Fogarty T, Jack A, Sumanaweera T, Maguire P: Noninvasive stereotactic radiosurgery (CyberHeart) for creation of ablation lesions in the atrium. Heart Rhythm. 2010, 7:802-10. 10.1016/j.hrthm.2010.02.010

6. Chang SD, Main W, Martin DP, Gibbs IC, Heilbrun MP: An analysis of the accuracy of the CyberKnife: a robotic frameless stereotactic radiosurgical system. Neurosurg. 2003, 52:140-6.

7. Lim M, Villavicencio AT, Burneikiene S, Chang SD, Romanelli P, McNeely L, McIntyre M, Thramann JJ, Adler JR: CyberKnife radiosurgery for idiopathic trigeminal neuralgia . Neurosurg Focus. 2005, 18:E9.

8. Zei PC, Soltys SG, Loo B, et al.: First-in-man treatment of arrhythmia (ventricular tachycardia) using stereotactic radiosurgery. Heart Rhythm. 2013, 10:1-554.

9. Sacher F, Roberts-Thomson K, Maury P, Tedrow U, Nault I, Steven D, Hocini M, Koplan B, Leroux L, Derval N, Seiler J, Wright MJ, Epstein L, Haissaguerre M, Jais P, Stevenson WG: Epicardial ventricular tachycardia ablation a multicenter safety study . J Am Coll Cardiol. 2010, 55:2366-72. 10.1016/j.jacc.2009.10.084

10. Josephson ME, Harken AH, Horowitz LN: Endocardial excision: a new surgical technique for the treatment of recurrent ventricular tachycardia. Circulation. 1979, 60:1430-9.

11. Hoogeman M, Prévost JB, Nuyttens J, Pöll J, Levendag P, Heijmen B: Clinical accuracy of the respiratory tumor tracking system of the CyberKnife: Assessment by analysis of log files. Int J Radiat Oncol Biol Phys. 2009, 74:297-303. 10.1016/j.ijrobp.2008.12.041

12. Blanck O, Bode F, Gebhard M, Hunold P, Brandt S, Bruder R, Grossherr M, Vonthein R, Rades D, Dunst J: Dose-escalation study for cardiac radiosurgery in a porcine model . Int J Radiat Oncol Biol Phys. 2014, 89:590-8. 10.1016/j.ijrobp.2014.02.036 